\title{
Analysis of Crystal Lattice Deformation by Ion Channeling
}

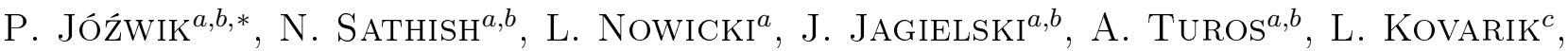

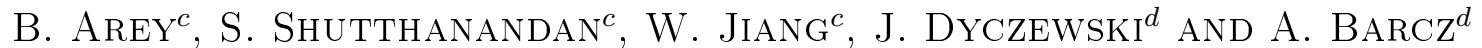 \\ ${ }^{a}$ National Centre for Nuclear Research, A. Sołtana 7, 05-400 Otwock/Świerk, Poland \\ ${ }^{b}$ Institute for Electronic Materials Technology, Wólczyńska 133, 01-919 Warszawa, Poland \\ ${ }^{c}$ Pacific Northwest National Laboratory, Richland, WA 99352, USA \\ ${ }^{d}$ Institute of Physics, PAS, al. Lotników 32 /46, 02-668 Warszawa, Poland
}

\begin{abstract}
A model of dislocations has been developed for the use in Monte Carlo simulations of ion channeling spectra obtained for defected crystals. High resolution transmission electron microscopy micrographs show that the dominant type of defects in the majority of ion irradiated crystals are dislocations. The RBS/channeling spectrum is then composed of two components: one is due to direct scattering on randomly displaced atoms and the second one is related to beam defocussing on dislocations, which produce predominantly crystal lattice distortions, i.e. bent channels. In order to provide a correct analysis of backscattering spectra for the crystals containing dislocations we have modified the existing Monte Carlo simulation code "McChasy". A new version of the code has been developed by implementing dislocations on the basis of the Peierls-Nabarro model. Parameters of the model have been determined from the high resolution transmission electron microscopy data. The newly developed method has been used to study the Ar-ion bombarded $\mathrm{SrTiO}_{3}$ samples. The best fit to the Rutherford backscattering/channeling spectra has been obtained by optimizing the linear combination of two kinds of defects: displaced atoms and bent channels. The great virtue of the Monte Carlo simulation is that unlike a traditional dechanneling analysis it allows quantitative analysis of crystals containing a mixture of different types of defects.
\end{abstract}

DOI: $10.12693 /$ APhysPolA.123.828

PACS: 61.85.+p, 68.55.Ln, 02.70.Uu, 68.37.Og

\section{Introduction}

Strontium titanate $\left(\mathrm{SrTiO}_{3}, \mathrm{STO}\right)$ is a material occurring in a perovskite structure. It has outstanding properties like a high melting point ( $2353 \mathrm{~K}$ ), high mechanical strength and high thermal stability. Thin layers of STO have been used in electronics. The most recent research reveals that STO may be suitable also in nuclear power engineering. Jiang et al. have shown that it is a good candidate for a waste form that is used for disposal of radioactive wastes generated in nuclear power plants [1]. It is also presumed that STO can be used as an inert matrix for incorporating a fissile phase in a new generation of nuclear fuels. For that purpose it is necessary to learn what sort of defects are present in the STO structure as well as what is their formation mechanism.

The Rutherford backscattering (RBS) measurements in ion channeling orientation have been widely used to study defects in crystals. Analysis of RBS spectra obtained for single-element crystals has been based on the method proposed by Bøgh called the two beam approximation (TBA) [2]. This approach assumes that there is one type of defect present which is an amorphous phase and that there are two processes having an effect on the spectra: the first one is scattering due to lattice defects and the other one is beam defocusing when the analyzing

*corresponding author; e-mail: przemyslaw.jozwik@itme.edu.pl particles travel through the damaged layer. TBA analysis allows to obtain defect depth-distributions.

The RBS spectra obtained for complex crystals are distinct from those obtained for the single-element materials owing to the presence of more than one sublattice. In that case it becomes difficult to determine the background dechanneling required in the TBA approach as well as the depth distribution of the defects. Moreover, the TBA method is unable to take into account extended defects like dislocations or stacking faults. The proper deconvolution procedure should thus consider kinds of defects present in the damaged crystals, for this input from the complementary technique permitting the identification of defects (e.g. high resolution transmission electron microscopy, HRTEM) is needed in order to analyze the RBS/channeling (RBS/C) spectra correctly.

In 1971 Barret postulated the Monte Carlo (MC) simulations as a promising method of RBS analysis [3]. Indeed, they allow depth distributions of defects to be calculated. We have performed MC simulations using a simulation code called McChasy $[4,5]$. The use of HRTEM helps to improve the simulation process because it facilitates determination of types of defects present in a crystal and enables measurements of their geometry.

In this paper we report progress in the development of the McChasy code that has been modified to include the dechanneling process on dislocations, described accordingly to the Peierls-Nabarro model [6, 7]. Previous modifications of the code were described in Refs. [8] and [9]. Application of McChasy for simulating the RBS/C spectra of the STO irradiated crystals has allowed us to de- 
termine depth-distributions of defect types that are dominant for this structure. It was also a test that has told us whether changes in the McChasy code are implemented properly and we briefly describe the results in this paper.

\section{Experimental}

STO single crystals, $0.5 \mathrm{~mm}$ in thickness, were irradiated with $320 \mathrm{keV} \mathrm{Ar}^{+}$ions up to a fluence ranging from $1 \times 10^{14} \mathrm{~cm}^{-2}$ to $5 \times 10^{15} \mathrm{~cm}^{-2}$. Implantation was performed at room temperature (RT) in the Institute of Electronic Materials Technology. The $\mathrm{Ar}^{+}$ion current was below $1 \mu \mathrm{A}$ per $\mathrm{cm}^{2}$ in order to avoid excessive sample heating. HRTEM measurements were performed in the Environmental Molecular Sciences Laboratory (EMSL) at Pacific Northwest National Laboratory. RBS measurements were performed in the EMSL (at energies of $1.4,2.2$ and $2.9 \mathrm{MeV}$ ) and in the Institute of Physics at the Polish Academy of Sciences (at an energy of $2.0 \mathrm{MeV}$ ).

A cross-sectional HRTEM investigation was performed using a FEI Titan HRTEM operated at $300 \mathrm{kV}$. For the RBS analysis the STO crystals were oriented along the (0001) axis. The RBS/C spectra were measured for virgin (i.e. non-implanted) and irradiated samples with a ${ }^{4} \mathrm{He}$ ion beam at energies varying from 1.4 up to $2.9 \mathrm{MeV}$. The spectra were fitted by the Monte Carlo simulation code (McChasy).

\section{Modification of the simulation procedure}

"McChasy" is a short for Monte Carlo channeling symulation code. It has been developed and designed for analyzing the RBS experimental spectra obtained in channeling conditions [4]. McChasy is able to calculate RBS spectra for light-ions in a variety of crystalline targets. During the simulation process McChasy creates a virtual crystal but considers always a single cell. The cell is divided into a few tens of virtual slices. McChasy calculates the trajectory of an impinging ion slice by slice, allowing for the interaction between the ion and the atoms in the cell. The spectra obtained for defected structures can be also fitted by the McChasy code. McChasy simply changes the coordinates of some target atoms in a random way and then calculates their interaction with the analyzing ion.

The first approach to reproduce scattering on dislocations was based on the assumption that every dislocation can be considered as a bent channel (BC). The bending angle $\eta$, i.e. the angle between the direction determined by the straight channels and a tangent line at the inflexion point, was set equal to $1^{\circ}$. The HRTEM micrograph analysis (after an inverse Fourier transformation was applied) has revealed that an extra plane in a crystal causes bending of a series of neighbouring channels (cf. Fig. 1). The maximum bending angle measured for the most bent channel reaches the values up to $14^{\circ}$ for STO. Moreover, the angle as well as the shift of a channel produced by the extra plane both decrease with the distance from a dislocation core.

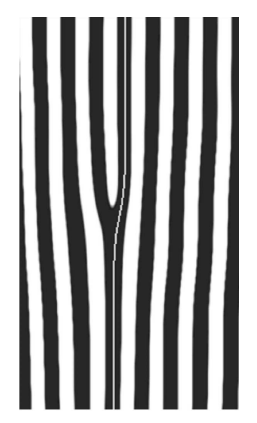

Fig. 1. Inverse Fourier transform of an HRTEM micrograph of STO irradiated crystal showing a series of bent channels in the vicinity of dislocation and the arctan function fitted to the bent channel.

According to the Peierls-Nabarro model the shape of $\mathrm{BC}$ can be fitted by the arctan function $[6,7]$ :

$u(z)=b \arctan (a z)$,

where $b$ and $a$ are the fitting parameters that can be obtained from the HRTEM micrographs. Parameter $b$ is closely connected with the shift of the BCs. It can also be easily shown that

$\tan \eta=a b$.

We obtained the dependence of the bending angle and the parameter $b$ on the distance from the dislocation core for the STO structure

$$
\begin{aligned}
& \eta(r)=11^{\circ} \exp \left(-\frac{r}{2500}\right), \\
& b(r)=12.25 \exp \left(-\frac{r}{5000}\right),
\end{aligned}
$$

where $r$ is the distance from the dislocation core expressed in pm.

Based on these results we have modified the McChasy code in order to provide more accurate calculations. In the current version of the code the arctan function as well as the dependences $\eta(r)$ and $b(r)$ are used to parameterize $\mathrm{BCs}$ formed in the vicinity of dislocations. At the beginning of the simulation process McChasy reads the depth distribution of BCs provided by the user, picks random coordinates of dislocation cores in the virtual crystal and calculates displacements of target atoms where needed. Then it calculates the trajectory of the ion as described above.

McChasy is probably the only simulation code that is able to simulate both backscattering from randomly displaced atoms and the dechanneling process on dislocations. The code is able to analyze data recorded for crystals with relatively low density of dislocations. Burgers vectors of dislocations modeled for McChasy make a straight angle to the direction of the analyzing ion beam.

\section{Results and discussion}

For the sake of clarity in Fig. 2 there are shown only RBS spectra recorded at energy $2.0 \mathrm{MeV}$ for the STO 
crystal implanted to a fluence of $3 \times 10^{14} \mathrm{~cm}^{-2}$. Note that the additional RBS spectra were measured and simulated at the energies of $1.4,2.2$, and $2.9 \mathrm{MeV}$ for the samples irradiated to other fluences mentioned before in order to confirm whether the obtained depth-distributions of defects obtained are correct. MC simulations have been provided with two different procedures: the first one (henceforth denoted RDA) on the assumption that randomly displaced atoms are the only type of defect present in the structure and the other one (henceforth denoted $\mathrm{RDA}+\mathrm{BC}$ ) on the assumption of coexistence of both RDA and dislocations causing channel bending which decreases with the distance from the dislocation cores.

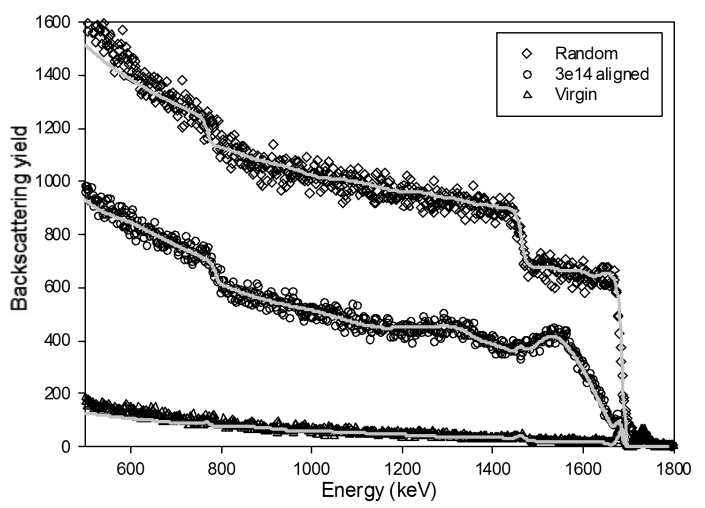

Fig. 2. RBS/C spectra recorded at an energy of $2.0 \mathrm{MeV}$ for a STO crystal irradiated with $320 \mathrm{keV} \mathrm{Ar}^{+}$ ions up to a fluence of $3 \times 10^{14} \mathrm{~cm}^{-2}$. Solid gray lines denote spectra fitted with the Monte Carlo simulation code.

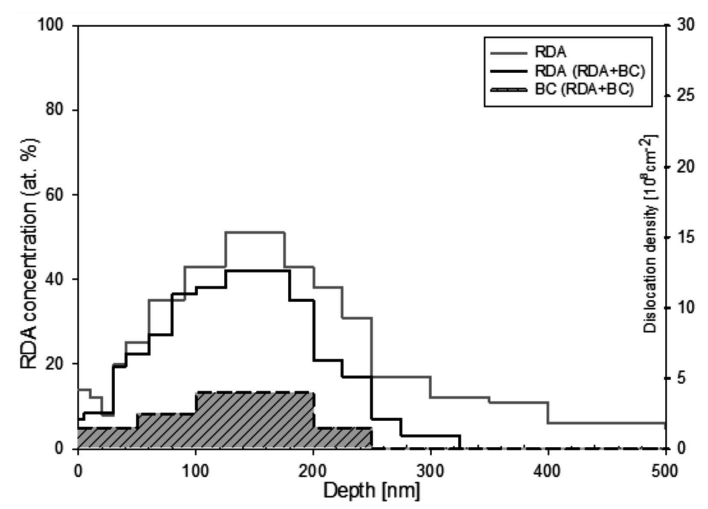

Fig. 3. Depth distribution of defects obtained from the Monte Carlo simulations.

The results of the MC analysis are the depth-distributions of defects shown in Fig. 3. The concentration of RDA is expressed in atomic \% since the density of dislocations is expressed in $10^{8} \mathrm{~cm}^{-2}$. Simulations provided with RDA only seem to lead to a non-physical solution. There is a tail seen in the defect distribution plot for the RDA procedure extending far away above the range of $320 \mathrm{keV}$ Ar ions in $\operatorname{STO}\left(R_{\mathrm{p}} \approx 168 \mathrm{~nm}\right.$, $\left.\Delta R_{\mathrm{p}} \approx 46 \mathrm{~nm}\right)$. The reason for this is an incorrect assumption of defect types. Apparently, dislocations are numerously present in the STO defected crystals and amorphous-like structures are not the dominant type of defect. Simulations based on the $\mathrm{RDA}+\mathrm{BC}$ procedure eliminate this tail and, as expected, the concentration of the RDA component decreases when the $\mathrm{BC}$ component is taken into consideration in the simulation procedure.

\section{Conclusions}

The current status of the Monte Carlo simulation code (McChasy) allows a fair reproduction of the dechanneling process on dislocations. The code uses the geometry of bent channels formed due to the presence of dislocations in the structure. The Monte Carlo simulations let us learn about defected structures and predict their behaviour when exposed to radiation. RBS/C and HRTEM turn out to be the complementary methods of analyzing defected structures and the use of the McChasy code makes it a very promising tool to determine cross-sections for formation of dislocations as well as precise depth-distributions of defects in complex crystals.

\section{Acknowledgments}

This work was supported by the research grant from the Polish Ministry of Science and Higher Education number $714 /$ N-EMSL/2010/0 and the Nuclear Energy Research \& Development, U.S. Department of Energy under Contract DE-AC05-76RL01830 and the EMSL Open Access project 34930. The research was performed using EMSL, a national scientific user facility sponsored by the Department of Energy's Office of Biological and Environmental Research and located at Pacific Northwest National Laboratory.

\section{References}

[1] W. Jiang, M.E. Bowden, Z. Zhu, P. Jóźwik, J. Jagielski, A. Stonert, Ind. Eng. Chem. Res. 51,621 (2012).

[2] E. Bøgh, Can. J. Phys. 46, 653 (1968).

[3] J.H. Barret, Phys. Rev. B 3, 1527 (1971).

[4] A. Dygo, A. Turos, Phys. Rev. B 40, 7704 (1989).

[5] L. Nowicki, A. Turos, R. Ratajczak, A. Stonert, F. Garrido, Nucl Instrum. Methods Phys. Res. B 240, 277 (2005).

[6] R. Peierls, Proc. Phys. Soc. 52, 34 (1940).

[7] F.R.N. Nabarro, Proc. Phys. Soc. 59, 256 (1947).

[8] A. Turos, L. Nowicki, A. Stonert, K. Pagowska, J. Jagielski, A. Muecklich, Nucl. Instrum. Methods Phys. Res. B 268, 1718 (2010).

[9] J. Jagielski, L. Thomé, Y. Zhang, C.M. Wang, A. Turos, L. Nowicki, K. Pagowska, I. Jozwik, Nucl. Instrum. Methods Phys. Res. B 268, 2056 (2010). 